\title{
Augmented EFIE with Adaptive Cross Approximation Algorithm for Analysis of Electromagnetic Problems
}

\author{
D. Z. Ding, Y. Shi, Z. N. Jiang, and R. S. Chen \\ Department of Communication Engineering, Nanjing University of Science and Technology, Nanjing 210094, China \\ Correspondence should be addressed to D. Z. Ding; dzding@mail.njust.edu.cn
}

Received 8 July 2013; Revised 15 October 2013; Accepted 27 October 2013

Academic Editor: Jan Fostier

Copyright (C) 2013 D. Z. Ding et al. This is an open access article distributed under the Creative Commons Attribution License, which permits unrestricted use, distribution, and reproduction in any medium, provided the original work is properly cited.

\begin{abstract}
The augmented electric field integral equation (A-EFIE) with charge neutrality enforcement provides a stable formulation to conquer low-frequency breakdown characteristic of conventional EFIE. It is augmented with additional charge unknowns through current continuity equation. The A-EFIE combined with the multilevel adaptive cross-approximation (MLACA) algorithm is developed to further reduce the memory requirement and computation time for analyzing electromagnetic problems. Numerical examples are given to demonstrate the accuracy and efficiency of the proposed method.
\end{abstract}

\section{Introduction}

Electromagnetic integral equations are often discretized with the method of moments (MoM) $[1,2]$, one of the most widespread and generally accepted techniques for electromagnetic problems. The integral equation is first discretized into a matrix equation using the Galerkin-based MoM with subdomain basis functions such as rooftop functions [3] for curvilinear quad patches and Rao-Wilton-Glisson (RWG) functions [4] for triangular patches. It is convenient to model objects with arbitrary shape using triangular patches; hence, RWG functions are widely used for representing unknown current distributions. When iterative solvers are used to solve the MoM matrix equation, the fast multipole algorithm (FMA) or multilevel fast multipole algorithm (MLFMA) [58] can be used to accelerate the calculation of matrix-vector multiplies in iterative algorithm.

However, the electric field integral equation (EFIE) only works well at mid-frequencies due to the well-known lowfrequency breakdown. To circumvent this disadvantage, some methods have been proposed to remedy low-frequency characteristic of electrical field integral equation (EFIE) [912]. Wilton and Glisson proposed loop-tree methods for the Helmholtz decomposition feature at low frequency regime [10]. Mautz and Harrington proposed a loop-star method and analyzed the reason of instability for integral equation in lowfrequency regime [11]. Lim et al. introduced loop-star method into RWG functions [12]. Wu et al. provided the comparison between the loop-tree method and loop-star method for the EFIE [13]. However, the loop-star or loop-tree basis functions only partly remove the problem at very low frequencies. Furthermore, the resulting system matrix in MoM is still illconditioned leading to slowly converging iterative solutions. Zhao and Chew utilized a basis rearrangement technique [14] to speed up convergence speed of the iterative solver, but it is also time consuming.

A well-conditioned EFIE (WEFIE) to overcome the shortcomings of the EFIE has been proposed in [15-23]. Due to the fact that the square of the EFIE operator does not have eigenvalues accumulating at zero or infinity, the WEFIE can give rise to a well-conditioned EFIE system independently of the discretization density [15, 17-19]. An efficient Calderón preconditioner is introduced based on the Calderón identities for scattering by perfect electric conductor (PEC) $[16,20-23]$. The Calderón preconditioner is made purely multiplicative by using the Buffa-Christiansen (BC) basis functions. It has also been used to accelerate the convergence rate of the iterative solution of the Poggio-Miller-Chang-Harrington-Wu-Tsai (PMCHWT) equations for wave scattering by homogeneous dielectric objects [24]. 
Some other fast methods have also been proposed to cover the regime from low frequency to mid frequency, such as the plane wave methods based on the generalized Gaussian quadrature rules [25] and the low-frequency fast inhomogeneous plane wave algorithm (LF-FIPWA) [26]. Since evanescent waves are highly direction dependent, much memory is required for LF-FIPWA. The fast multipole algorithm is numerically unstable due to the oscillatory characteristic of the spherical Hankel function for small arguments. As an attempt for a possible remedy, the mixed-form fast multipole algorithm (MF-FMA) [27] is proposed to cover a wide band from the low frequency to mid frequency. However, it is noticed that the MF-FMA need to construct multipole expansions for each nonempty box at the low-frequency regime. This results in a source distribution of high-density for the whole object. The adaptive MF-FMA (AMF-FMA) is proposed for efficient analysis of electromagnetic scattering from objects containing fine structure [28]. The AMF-FMA applies MF-FMA for some parts of the object which belongs to the low-frequency regime and the rest of the object which belongs to the mid-frequency regime is still analyzed by MLFMA. Hence, for a cuboid with many fine structures in [28], AMF-FMA takes advantage of adaptive grouping scheme to set the finest level box size as $0.05 \lambda$ for fine structure, while the finest level box size is $0.2 \lambda$ for the rest of the object. Much memory can be saved for AMF-FMA compared to MLFMA.

The augmented electric field equation (A-EFIE) $[16,29-$ 33] method has been proposed to solve the low-frequency problem without the loop-tree decomposition. As a new fullwave method, A-EFIE provides a stable formulation over a wide frequency band. In A-EFIE, the contributions of the vector potential and the scalar potential are separated; thus the imbalance between them in the conventional EFIE is avoided. It is augmented with additional charge unknowns through current continuity equation. Qian and Chew emphasize the charge neutrality enforcement (CNE) to remove the rankdeficient probelm in A-EFIE [30]. The complex interconnect packaging problem with over one million unknowns is solved without the help of parallel computers. Qian and Chew also proposed a perturbation method for solving the possible low-frequency inaccuracy problem of A-EFIE [31]. Chen et al. extended the A-EFIE method into the layered medium problem [33]. Yan et al. proposed a Calderón preconditioned A-EFIE algorithm (CP-AEFIE) for analysis of scattering by electrically large objects [16]. The resonance problem of the Calderón preconditioned EFIE is removed by introducing a reasonable complex wavenumber into the Calderón preconditioner [16].

Though the Calderón preconditioning method can eliminate the low-frequency breakdown and the dense-grid breakdown, it is highly technical. For the MLFMA and its low-frequency extensions, however, a priori knowledge of Green's function is needed. As a result, it cannot be easily applied to analyze the layered media problems. Compared to the MLFMA, the multilevel adaptive crossapproximation (MLACA) algorithm [34-36] is another popular technique for analyzing scattering/radiation problems. It makes use of the well-known fact that the approximate rank of the submatrices is low when the subscatterers are sufficiently separated. The MLACA is purely algebraic and therefore does not depend on a priori knowledge of Green's function. In this paper, the MLACA algorithm is introduced into the A-EFIE with CNE to accelerate the matrix-vector multiplies in iterative algorithm. The ACA algorithm is an adaptive and on-the-fly rank-revealing block factorization of the rank-deficient submatrices. Compared to the singular value decomposition (SVD) technique, the ACA algorithm only requires partial knowledge of the original matrix. It has been shown that for moderate electrical size problems the memory and CPU time requirements for the ACA algorithm scale as $O\left(N^{4 / 3} \log N\right)$ [35]. However, the set-up time of the MLFMA is less than that of ACA due to the fact that the MLFMA reuses multipole and local expansion information across levels. Jiang et al. utilize the predetermined interaction list supported octree (PILOT) $[36,37]$ to reduce the set-up time and the memory consumption of the ACA algorithm.

The aim of this paper is to apply the MLACA algorithm to improve the efficiency of the A-EFIE with CNE for analyzing electromagnetic problems. This paper is organized as follows. Section 2 describes the theory and implementation of the A-EFIE combined with the ACA algorithm in more details. Section 3 gives some numerical examples to demonstrate the accuracy and efficiency of our approach. Section 4 gives some conclusions.

\section{A-EFIE with MLACA Algorithm}

2.1. A-EFIE Formulation. The conventional EFIE is given as

$$
\left(i k_{0} \eta_{0} \bar{V}+\frac{\eta_{0}}{i k_{0}} \bar{S}\right) \cdot j=\vec{b},
$$

where the $\eta_{0}$ is free space wave impedance and $k_{0}$ is the wavenumber. $\vec{b}$ denotes the right-hand vector (excitation). $\vec{j}$ is the unknown current. The $\bar{V}$ and $\bar{S}$ correspond to the magnetic vector potential and electric scalar potential:

$$
\begin{gathered}
{[\bar{V}]_{m, n}=\mu_{r} \cdot \int_{S} \vec{\rho}_{m}(\vec{r}) \cdot \int_{S^{\prime}} g\left(\vec{r}, \vec{r}^{\prime}\right) \cdot \vec{\rho}_{n}\left(\vec{r}^{\prime}\right) d S^{\prime} d S} \\
{[\bar{S}]_{m, n}=\varepsilon_{r}^{-1} \cdot \int_{S} \nabla \cdot \vec{\rho}_{m}(\vec{r}) \cdot \int_{S^{\prime}} g\left(\vec{r}, \vec{r}^{\prime}\right) \cdot \nabla^{\prime}} \\
\cdot \vec{\rho}_{n}\left(\vec{r}^{\prime}\right) d S^{\prime} d S,
\end{gathered}
$$

where the subscripts $m, n$ denote the interaction between the $m$ th line and the $n$th line. $g\left(\vec{r}, \vec{r}^{\prime}\right)$ denotes free space scalar Green's function. $\vec{\rho}(\vec{r})$ denotes RWG basis function. $\mu_{r}$ and $\varepsilon_{r}$ represent the relative permeability and the relative permittivity, respectively. In A-EFIE, we use a normalized RWG function to expand the surface current by removing the edge length from the original RWG basis function:

$$
\vec{\rho}_{m}(\vec{r})= \begin{cases}\frac{\vec{r}-\vec{r}_{m}^{+}}{2 A_{m}^{+}}, & \vec{r} \in T_{m}^{+} \\ \frac{\vec{r}-\vec{r}_{m}^{-}}{2 A_{m}^{-}}, & \vec{r} \in T_{m}^{-} \\ 0, & \text { otherwise }\end{cases}
$$


where the $A_{m}^{ \pm}$is the area of the triangle $T_{m}^{ \pm}$and $\vec{r}_{m}^{ \pm}$is the free vertices of the triangle pair. Then, we use pulse function to expand the charge on each element which is defined as follows:

$$
h_{m}(\vec{r})= \begin{cases}\frac{1}{A_{m}}, & \vec{r} \in T_{m} \\ 0, & \text { otherwise }\end{cases}
$$

The expansion equations of surface current and charge are given as

$$
\begin{gathered}
\vec{j}(\vec{r})=\sum_{m=1}^{n \text { Line }} j_{m} \vec{\rho}_{m}(\vec{r}), \\
q(\vec{r})=\sum_{m=1}^{n \text { Triangle }} \rho_{m} h_{m}(\vec{r}),
\end{gathered}
$$

where the $n$ Line is the number of inner lines $n$ Triangle and is the number of mesh elements. $j_{m}$ and $\rho_{m}$ are the unknown expansion coefficients for surface current and charge, respectively. According to the current continuity equation:

$$
\nabla \cdot \vec{j}(\vec{r})=i \omega q(\vec{r})
$$

we define a transform matrix $\bar{D}$ as follow [29]:

$$
\begin{aligned}
& {[\bar{D}]_{m, n}} \\
& = \begin{cases}0, & \text { patch } m \text { does not belong to RWG } n \\
1, & \text { patch } m \text { is the positive part of the RWG } n \\
-1, & \text { patch } m \text { is the negative part of the RWG } n .\end{cases}
\end{aligned}
$$

Then, the scalar potential matrix $\bar{S}$ can be factorized as

$$
\bar{S}=\bar{D}^{T} \cdot \bar{P} \cdot \bar{D}
$$

where the $\bar{P}$ denotes a patch-to-patch scalar potential matrix as

$$
[\bar{P}]_{m, n}=\varepsilon_{r}^{-1} \int_{S} h_{m}(\vec{r}) \int_{S^{\prime}} g\left(\vec{r}, \vec{r}^{\prime}\right) h_{m}\left(\vec{r}^{\prime}\right) d S^{\prime} d S .
$$

And we substitute (5) and (6); into (7) we get

$$
\bar{D} \cdot \vec{j}(\vec{r})=i k c_{0} q(\vec{r}),
$$

where the $c_{0}$ is the light speed in vacuum. Substituting the above equations into the EFIE matrix equation and enforcing the current continuity equation explicitly, we can arrive at the following A-EFIE system. So, we get the form of A-EFIE as

$$
\left(\begin{array}{cc}
\bar{V} & \bar{D}^{T} \cdot \bar{P} \\
\bar{D} & k_{0}^{2} \bar{I}
\end{array}\right) \cdot\left(\begin{array}{c}
i k_{0} \cdot \vec{j}_{m} \\
c_{0} \cdot q
\end{array}\right)=\left(\begin{array}{c}
\eta_{0}^{-1} \cdot \vec{b} \\
0
\end{array}\right)
$$

where the $\bar{I}$ is an identity matrix with dimension of $n$ Triangle.
At very low frequencies, charge neutrality causes rank deficiency in the above forms of the A-EFIE [30]. Therefore, we drop a charge unknown from each unattached objects due to the charge neutrality. Then, the vector of charge is reduced to be $\rho_{r} . r$ is the dimension of reduced charge vector $\rho_{r}$ and equal to $n$ Triangle $-t$ ( $t$ denotes the number of disconnected objects for a problem). We define two mapping matrixes $\bar{F}$ and $\bar{B}$ :

$$
\rho_{r}=\bar{F} \cdot \rho, \quad \rho=\bar{B} \cdot \rho_{r}
$$

where the $\rho$ represents the previous charge density coefficient in (6). The matrix $\bar{F}$ maps the full vector forward to the reduced one, and the matrix $\bar{B}$ projects the reduced vector backward to the full one. Both of them are highly sparse matrices. Then, the AEFIE in (12) is given as

$$
\left(\begin{array}{cc}
\bar{V} & \bar{D}^{T} \cdot \bar{P} \cdot \bar{B} \\
\bar{F} \cdot \bar{D} & k_{0}^{2} \cdot \bar{I}{ }_{r}
\end{array}\right) \cdot\left(\begin{array}{c}
i k_{0} \cdot j_{m} \\
c_{0} \cdot \rho_{r}
\end{array}\right)=\left(\begin{array}{c}
\eta_{0}^{-1} \cdot \vec{b} \\
0
\end{array}\right),
$$

where the $I_{r}$ is an identity matrix with dimension of $(n$ Triangle $-t) \times(n$ Triangle $-t)$. The linear system of equations in (14) can be solved by the restart GMRES iterative method with preconditioning technique to accelerate the convergence of the iterative solver $[5,6,31]$. A simple and efficient preconditioning matrix has been given as [31]

$$
\bar{M}=\left(\begin{array}{cc}
\operatorname{diag}(\bar{V})(\bar{F} \cdot \bar{D})^{T} \cdot\left(\bar{F} \cdot \operatorname{diag}(\bar{P}) \cdot \bar{F}^{T}\right) \\
\bar{F} \cdot \bar{D} & k_{0}^{2} \cdot \bar{I}_{r}
\end{array}\right),
$$

where the $\operatorname{diag}(\bar{V})$ is the diagonal matrix of $\bar{V}$. Since $\bar{M}$ is a sparse matrix, its inverse matrix can be solved by fast sparse matrix solver such as UMFPACK solver in this paper.

For the analysis of microstrip antenna and microwave integrated circuit (MIC) in layered medium, free space scalar Green's function $g\left(\vec{r}, \vec{r}^{\prime}\right)$ in (2) should be substituted by layered media Green's function [38]. In general, spatial domain Green's functions are expressed in terms of Sommerfeld integrals. Due to the highly oscillatory nature of the integrand, numerical integration is very time consuming. In this paper, a two-level generalized pencil of function (GPOF) method is utilized to realize DCIM [39]. Then, spatial domain Green's functions can be obtained in closed forms from their spectral-domain counterparts via the Sommerfeld identity.

2.2. MLACA Acceleration. The MLACA employs the same octree data structure as in the MLFMA [7]. The octal-tree algorithm is used to subdivide a box that encloses an object into smaller boxes. Figure 1 shows the decomposition of the problem domain at different levels. With reference to Figure 1, far interactions exit at levels 2 and higher. Far interactions can be computed using the MLACA [40].

According to the above octree data structure, the impedance matrix in (14) can be decomposed into near-field interactions $Z_{N}$ and far-field interactions $Z_{F}$. It can be simply written as

$$
[Z] \approx\left[Z_{N}\right]+\left[Z_{F}\right],
$$




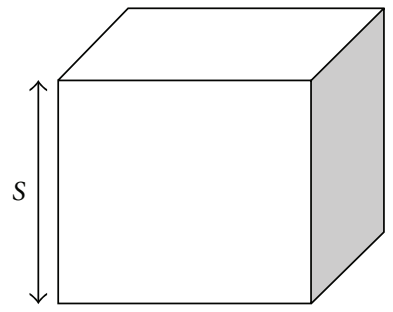

Level 0

(a)

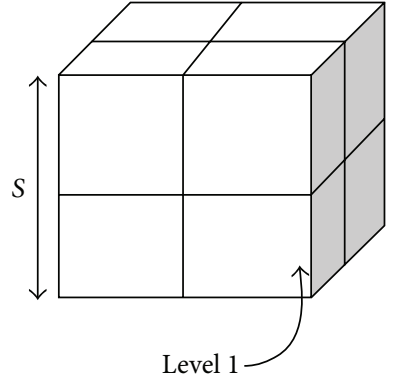

(b)

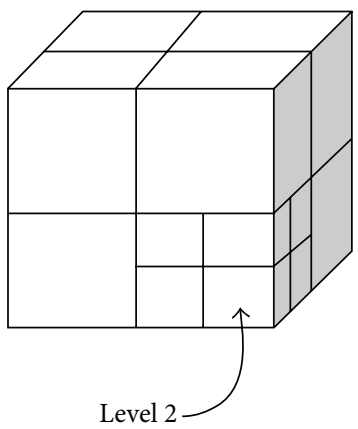

(c)

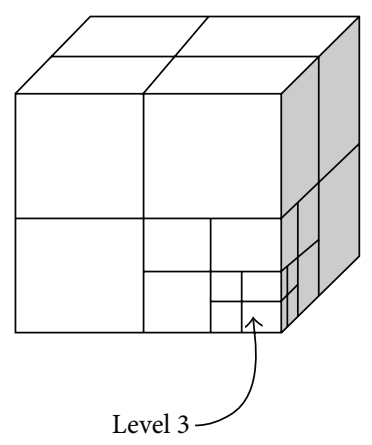

(d)

FIGURE 1: The sketch of the octree structure.

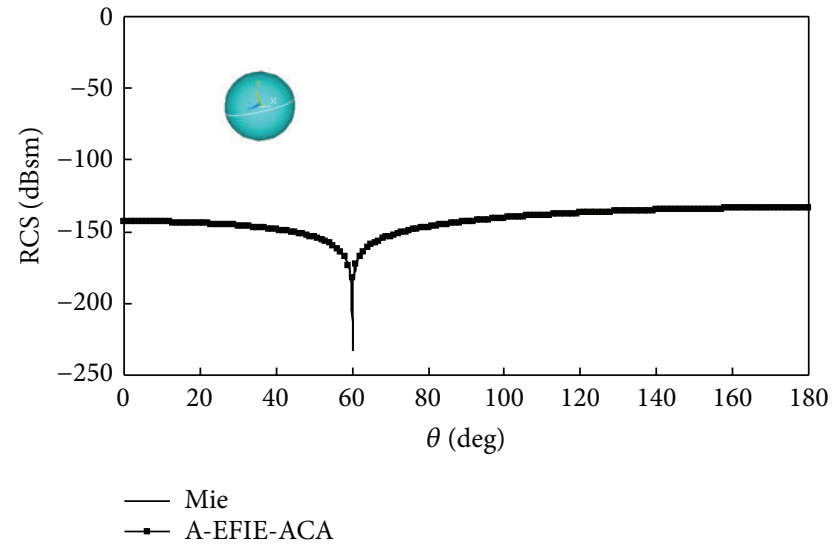

FIgURE 2: The VV-polarization bistatic RCS for PEC sphere at $150 \mathrm{KHz}$.

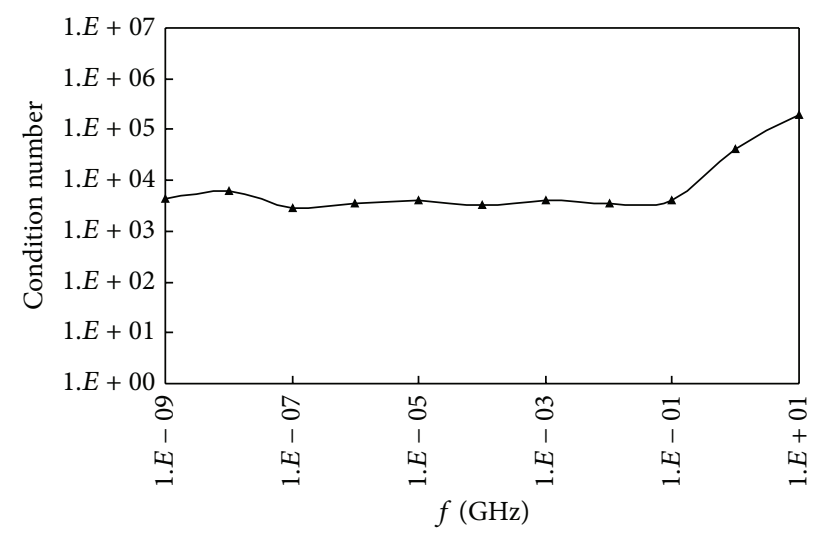

FIGURE 3: The condition number versus frequency for the A-EFIE with charge neutrality enforcement for the PEC sphere.

where the $Z_{N}$ and $Z_{F}$ are computed directly and compressed by the MLACA algorithm, respectively. When the two boxes are sufficiently separated, the impedance matrix associated with them can be expressed using low-rank representations.

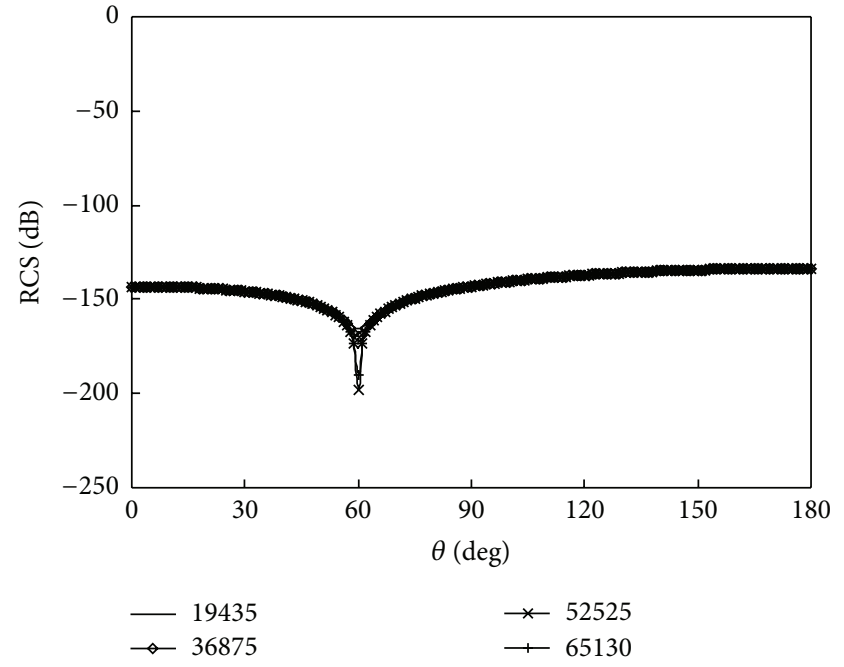

FIgURE 4: The VV-polarization bistatic RCS for PEC sphere with respect to the number of unknowns at $150 \mathrm{KHz}$.

This feature is utilized by the MLACA. In the MLACA implementation, the impedance matrix between two sufficiently distant boxes can be expressed in terms of two small matrices

$$
\left[Z_{i j}\right]_{m_{1} m_{2}} \approx\left[U_{i j}\right]_{m_{1} r}\left[V_{i j}\right]_{r m_{2}}^{H}
$$

where the $\left[Z_{i j}\right]_{m_{1} m_{2}}$ is the interaction matrix between the observation and source boxes. $m_{1}$ and $m_{2}$ denote the number of the basis functions in the observation and source boxes, respectively. The index $r$ denotes the rank of $\left[Z_{i j}\right]_{m_{1} m_{2}}$ and is much smaller than $m_{1}$ and $m_{2}$. The impedance matrix in (16) can be rewritten in multilevel operations as

$$
[Z] \approx\left[Z_{N}\right]+\sum_{l=2}^{L} \sum_{i=1}^{M(l)} \sum_{j=1}^{\operatorname{Far}(l(i))}\left[U_{l i j}\right]\left[V_{l i j}\right]^{H}
$$

where the $M(l)$ is the number of nonempty groups at level $l$ and $\operatorname{Far}(l(i))$ denotes the number of far interaction groups of the $i$ th nonempty group for each observer group $l(i)$ at level $l$. Since the matrices $[U]$ and $[V]$ generated by the MLACA are 
usually not orthogonal, they may contain redundancies that can be removed by the SVD technique [41].

Finally, the linear system of equations in (14) can be solved by preconditioned restated generalized minimal residual algorithm (GMRES) using the MLACA to accelerate the matrix-vector multiplies in iterative algorithm.

\section{Numerical Experiments}

In this section, we show some numerical results for electromagnetic structures that illustrate the effectiveness of the proposed A-EFIE with CNE accelerated by the MLACA algorithm. All numerical experiments are performed on Intel(R) Core (TM) 2 Quad CPU at $2.83 \mathrm{GHz}$ and $3.5 \mathrm{~GB}$ of RAM in double precision and the truncating tolerance of the ACA is $10^{-3}$ (relative to the largest singular value). The restart number of the generalized minimal residual (GMRES) is set to be 30. The inner-outer flexible GMRES algorithm (FGMRES) is used as the iterative solver for microstrip circuit problems.

Additional details and comments on the implementation are given below:

(i) zero vector is taken as initial approximate solution for all examples and all systems in each example;

(ii) the iteration process is terminated when the normalized backward error is reduced by $10^{-4}$ for all the examples.

First, electromagnetic scattering from a perfect electric conducting (PEC) sphere is analyzed in order to demonstrate the advantages of the proposed A-EFIE with the ACA algorithm (denoted as A-EFIE-ACA). The sphere has a radius of $2 \mathrm{~m}$. It is discretized with 7332 triangular patches leading to 18330 unknowns. The angle of incident plane wave is $\theta^{i}=0^{0}$, and $\phi^{i}=0^{0}$ with the frequency $f=150 \mathrm{KHz}$. Figure 2 shows the VV-polarization bistatic RCS solved by Mie series and A-EFIE-ACA. It can be found that the A-EFIE-ACA gives right results at low frequency. The condition numbers versus frequency are also demonstrated in Figure 3. It is observed from Figure 3 that the A-EFIE with charge neutrality enforcement makes the condition number almost constant when decreasing the frequency to $1 \mathrm{~Hz}$. The condition number grows slowly when increasing the frequency from $1 \mathrm{GHz}$ to $10 \mathrm{GHz}$. Figure 4 further shows the VV-polarization bistatic RCS when the number of unknowns for the above PEC sphere becomes larger. It can be found that the proposed A-EFIE-ACA works well when the number of unknowns increases from 19435 to 65130 . However, when the number of unknowns is increased to 76480, the procedure for A-EFIEACA cannot work due the dense-grid breakdown problem. Table 1 shows the comparison of memory requirement and solution time of GMRES between the A-EFIE-ACA and AEFIE without the ACA algorithm (denoted as A-EFIE-MoM) in bistatic RCS computation for the sphere. The 2-level ACA is used to reduce the solution time and memory requirement in A-EFIE-ACA. It can be found that the A-EFIE-ACA can
TABLE 1: The memory requirement and solution time in bistatic RCS computation for the sphere.

\begin{tabular}{lccc}
\hline Method & Memory & Solution time & $\begin{array}{c}\text { Number of levels } \\
\text { for ACA }\end{array}$ \\
\hline A-EFIE-MoM & $1332 \mathrm{MB}$ & $72 \mathrm{~s}$ & N/A \\
A-EFIE-ACA & $169 \mathrm{MB}$ & $24 \mathrm{~s}$ & 2 \\
\hline
\end{tabular}

TABLE 2: The memory requirement and solution time in bistatic RCS computation for the almond.

\begin{tabular}{lccc}
\hline Method & Memory & Solution time & $\begin{array}{c}\text { Number of levels } \\
\text { for ACA }\end{array}$ \\
\hline A-EFIE-MoM & $674 \mathrm{MB}$ & $178 \mathrm{~s}$ & N/A \\
A-EFIE-ACA & $228 \mathrm{MB}$ & $84 \mathrm{~s}$ & 2 \\
EFIE-ACA & $156 \mathrm{MB}$ & $556 \mathrm{~s}$ & 2 \\
\hline
\end{tabular}

save much time by a factor of 7.9 than that of the A-EFIEMoM. The A-EFIE-ACA can also save much solution time by a factor of 3.0 than that of the A-EFIE-MoM.

Next, we investigate the performance of the proposed AEFIE-ACA on electromagnetic scattering at high frequency. The mathematic description of NASA almond is defined in [42] and shown in Figure 5. The total length of the almond is 9.936 inches. The almond is discretized with 5216 triangular patches with 13040 unknowns. The angle of incident plane wave is $\theta^{i}=0^{0}$, and $\phi^{i}=0^{0}$. Figure 5 gives the VVpolarization bistatic RCS for an almond at $3 \mathrm{GHz}$. It can be found that the results obtained by the A-EFIE-ACA agree well with ones from traditional EFIE with ACA algorithm (denoted as EFIE-ACA). The 2-level ACA is used in the A-EFIE-ACA and EFIE-ACA. Table 2 shows the comparison of memory requirement and solution time of GMRES among different methods in bistatic RCS computation for the almond. It can be found that the A-EFIE-ACA can save much memory requirement by a factor of 2.1 than that of the A-EFIE-MoM, 6.6 than that of the EFIE- ACA. It can be also found that the A-EFIE-ACA can save much memory requirement by a factor of 2.9 than that of the A-EFIE-MoM. However, the memory requirement for the A-EFIE-ACA is more than that for the EFIE-ACA due to additional charge unknowns introduced into the A-EFIE.

The third example is a microstrip bandpass filter (BPF) based on five intercoupled split-ring resonators (SRR) proposed in [43]. The periodic SRR structure is shown in Figure 6. The distance between each SRR's arms is $0.05 \mathrm{~mm}$. The relative dielectric constant of substrate is 3.9. The structure is implemented on the substrate Corning 1059 with a relative dielectric constant of 3.9 and thickness of $0.37 \mathrm{~mm}$. Microstrip line width $w$ is $0.8 \mathrm{~mm}$. The width $d$ of the interdigital and the gap in each SRR structure are both $0.114 \mathrm{~mm}$. The length of each SRR structure is 0.5 wavelengths at center frequency of $12 \mathrm{GHz}$. The structure is discretized with 924 triangular patches with 1077 unknowns. Frequency is from $5 \mathrm{GHz}$ to $20 \mathrm{GHz}$. The sampled frequency interval is $0.1 \mathrm{GHz}$ and a total of 151 frequencies need to be calculated in the proposed A-EFIE-ACA. Figure 7 gives scattering parameters 


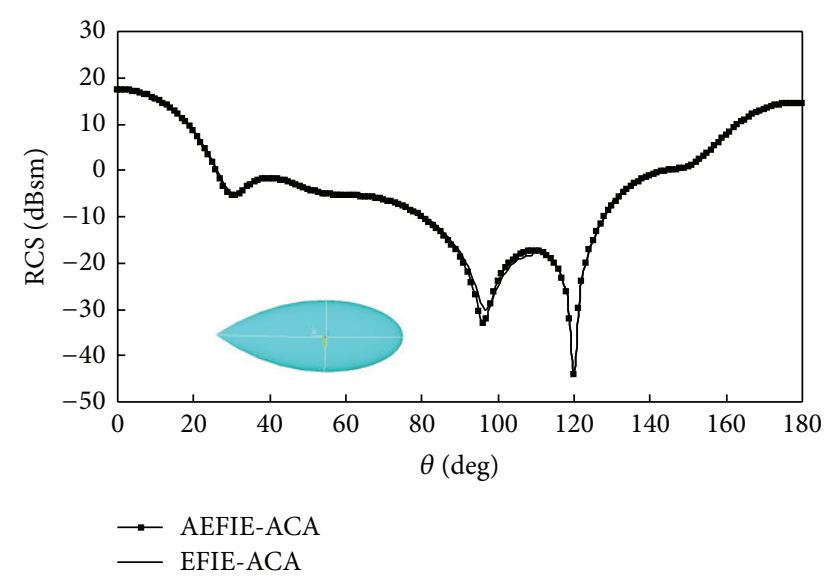

FIGURE 5: The VV-polarization bistatic RCS for an almond at $3 \mathrm{GHz}$.

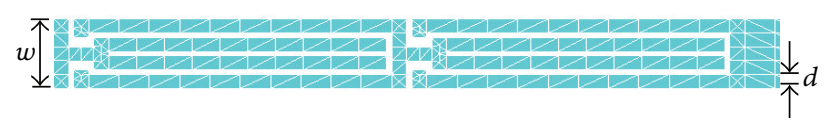

FIGURE 6: The periodic SRR structure in microstrip bandpass filter.

S11 and S12 obtained from the A-EFIE-ACA method for the above BPF structure. It can be found that the results from A-EFIE-ACA agree well with those from Ansoft Designer software. It is observed from Figure 6 that the BPF exhibits a passband region from 9.0 to $15.2 \mathrm{GHz}$, which corresponds to a fractional bandwidth of $49.6 \%$. The insertion losses in the passband region are around $1.2 \mathrm{~dB}$. Therefore, the simulated results confirm the feasibility of utilizing the intercoupled split-ring resonator structure in microwave bandpass filter applications. The BPF is a typical electrical small structure when the wavelength is $0.015 \mathrm{~m}$ at $20 \mathrm{GHz}$. The condition number of impedance matrix is very bad due to the fine structure in the BPF with SRR structure. Therefore, the inner-outer Flexible GMRES algorithm (FGMRES) is used as the iterative solver. In FGMRES, the near-field impedance matrix is used as the preconditioner in the inner iteration. In the FGMRES algorithm, the stop precision is 1.E-4. Table 3 shows the comparison of memory requirement and solution time among the A-EFIE-ACA, A-EFIE-MoM, and EFIE-ACA methods for the BPF with SRR structure. It can be found that the proposed A-EFIE-ACA can save much memory requirement by a factor of 2.54 than that of the A-EFIEMoM. The A-EFIE-ACA can also save much time for average solution time at each frequency by a factor of 1.48 than that of A-EFIE-MOM, by a factor of 39.4 than that of EFIE-ACA. Therefore, the A-EFIE-ACA can save much time for the total iterative solution time by a factor of 39.5 than that of the EFIEACA.

The last example is a dual-mode dual-band BPF [44] as shown in Figure 8. The dual-mode dual-band BPF is implemented on the substrate Duroid 6010 with a relative dielectric constant of 10.2 , loss tangent of 0.0023 , and thickness of $1.27 \mathrm{~mm}$. The related dimensions as shown in Figure 6 are determined as follows: $L_{1}=13.6 \mathrm{~mm}, L_{2}=3.8 \mathrm{~mm}$,

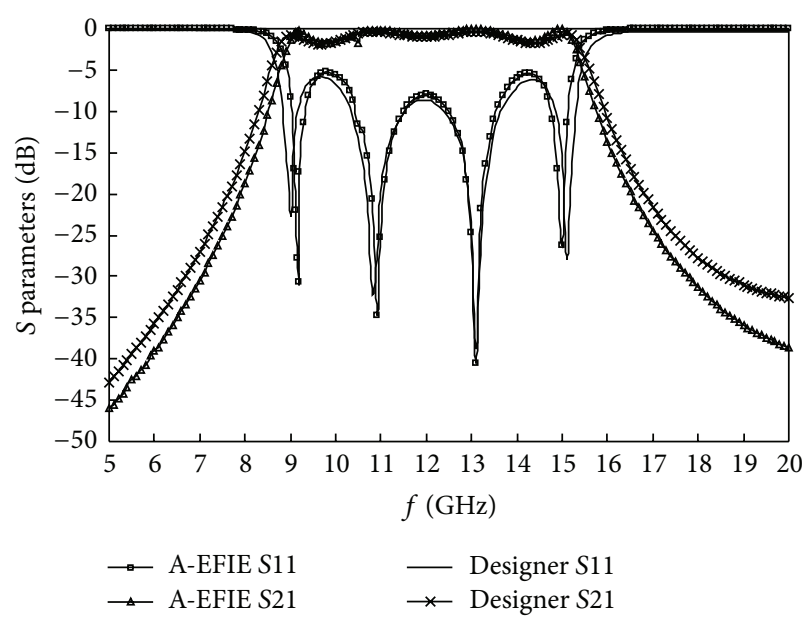

FIGURE 7: S11 and S12 for BPF with SRR structure.

TABLE 3: The memory requirement and solution time for BPF with SRR structure.

\begin{tabular}{lccc}
\hline Method & Memory & $\begin{array}{c}\text { Solution time for } \\
\text { each frequency }\end{array}$ & $\begin{array}{c}\text { Total solution } \\
\text { time }\end{array}$ \\
\hline A-EFIE-MoM & $28.2 \mathrm{MB}$ & $2.54 \mathrm{~s}$ & $385 \mathrm{~s}$ \\
A-EFIE-ACA & $11.1 \mathrm{MB}$ & $1.72 \mathrm{~s}$ & $259 \mathrm{~s}$ \\
EFIE-ACA & $7.7 \mathrm{MB}$ & $67.8 \mathrm{~s}$ & $10237 \mathrm{~s}$ \\
\hline
\end{tabular}

$L_{3}=2.6 \mathrm{~mm}, L_{4}=2.2 \mathrm{~mm}, W_{1}=0.9 \mathrm{~mm}, W_{2}=6 \mathrm{~mm}, W_{3}=$ $5.2 \mathrm{~mm}, W_{4}=6.4 \mathrm{~mm}$, and $W_{5}=1.2 \mathrm{~mm}$. The horizontal and vertical lengths of the slot are $9.2 \mathrm{~mm}$ and $6.2 \mathrm{~mm}$, respectively. The size of the patch including stubs is $0.46 \lambda_{g} \times$ $0.42 \lambda_{g}$, where $\lambda_{g}$ is $48 \mathrm{~mm}$, the $50 \Omega$ guided wavelength at the lower passband. The simulated results obtained from the A-EFIE-ACA method are given in Figure 9 compared to the results from Ansoft HFSS software. The BPF structure is discretized with 1476 triangular patches with 2070 unknowns. Frequency is from $1.5 \mathrm{GHz}$ to $6.0 \mathrm{GHz}$. The interval of frequency is $0.1 \mathrm{GHz}$. Good agreement is achieved between these two kinds of results. It is observed from Figure 8 that the lower and upper passbands have the fractional bandwidth of $12.8 \%$ and $6.5 \%$ centered at 2.3 and $5.05 \mathrm{GHz}$, respectively. The simulated insertion loss is $0.73 \mathrm{~dB}$ and $0.86 \mathrm{~dB}$, respectively. The return loss is better than $10 \mathrm{~dB}$. Two transmission zeros are generated near the passband edges, guaranteeing high selectivity. It also can be found that two transmission poles in both passbands demonstrate the effectiveness of the dualmode dual-band filter. The 3-level ACA is used. The SVD is used to recompress the far interaction matrix in the A-EFIEACA method. Table 4 shows the comparison of memory requirement and solution time among the A-EFIE-ACA, AEFIE-MoM, and EFIE-ACA methods for the BPF structure. It can be found that the proposed A-EFIE-ACA can save much memory requirement by a factor of 2.44 than that of the AEFIE-MoM. The average solution time at each frequency for the A-EFIE-MOM is about 1.34 times than that of of the AEFIE-ACA. Furthermore, the A-EFIE-ACA can save much time by a factor of 31.3 than that of the EFIE-ACA. It can 


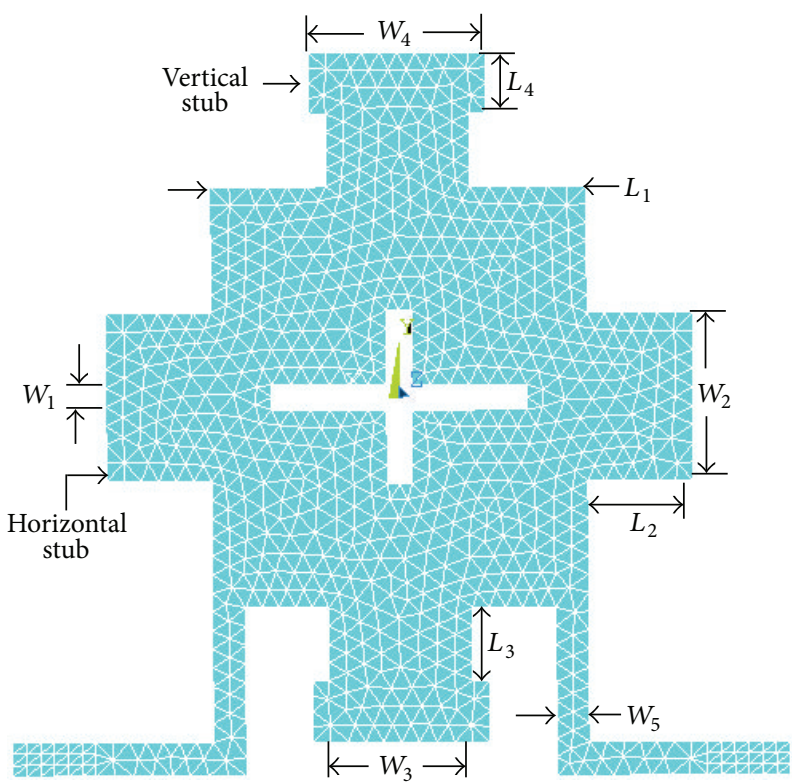

FIgure 8: Model of a dual-mode dual-band BPF.

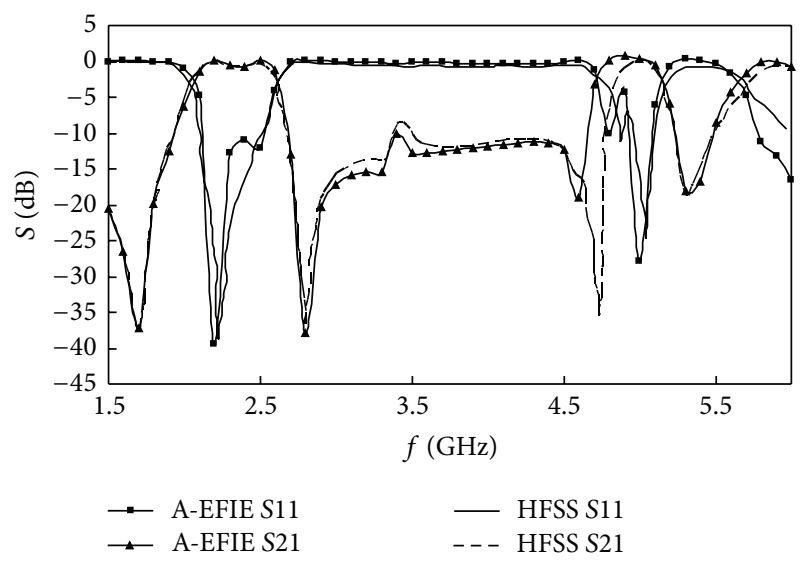

FIGURE 9: S11 and S12 for a dual-mode dual-band BPF.

TABLE 4: The memory requirement and solution time for a dualmode dual-band BPF.

\begin{tabular}{lccc}
\hline Method & Memory & $\begin{array}{c}\text { Solution time for } \\
\text { each frequency }\end{array}$ & $\begin{array}{c}\text { Total solution } \\
\text { time }\end{array}$ \\
\hline A-EFIE-MoM & $51.2 \mathrm{MB}$ & $7.38 \mathrm{~s}$ & $345 \mathrm{~s}$ \\
A-EFIE-ACA & $20.9 \mathrm{MB}$ & $5.47 \mathrm{~s}$ & $252 \mathrm{~s}$ \\
EFIE-ACA & $14.1 \mathrm{MB}$ & $171.3 \mathrm{~s}$ & $7879.3 \mathrm{~s}$ \\
\hline
\end{tabular}

be concluded that the proposed A-EFIE-ACA is very efficient compared to other methods.

\section{Conclusions}

In this paper, the A-EFIE with CNE accelerated by the MLACA algorithm is presented for solving electromagnetic scattering and microstrip circuit problems. Numerical experiments are performed and the comparison is made with the EFIE-ACA and A-EFIE-MoM methods. It can be found that the proposed A-EFIE-ACA method is more efficient and can significantly reduce the overall computational cost. Due to the fact that the set-up time of the ACA is much more than that of MLFMA, it is known that the ACA does not maintain its favorable complexity once the problem becomes electrically large. The PILOT algorithm can be used to reduce the set-up time and the memory consumption of ACA. The PILOT algorithm utilizes the idea that the farfield interaction lists of siblings share many common cubes to regroup a new far-field interaction list based on an octal tree data structure. Higher compression is achieved by using the PILOT algorithm when the dimension of the matrix is large. Further investigations deserve to be undertaken to study efficiency of the PILOT algorithm when the proposed AEFIE-ACA method is used for the analysis of electromagnetic problems from electrically large objects.

\section{Acknowledgments}

The authors would like to thank Jiangsu Natural Science Foundation (BK2012034) and Natural Science Foundation (61171041) for support.

\section{References}

[1] R. F. Harrington, Field Computation by Moment Methods, R. E. Krieger, Malabar, Fla, USA, 1968.

[2] D. Z. Ding and R. S. Chen, "Electromagnetic scattering by conducting bor coated with chiral media above a lossy halfspace," Progress in Electromagnetics Research, vol. 104, pp. 385401,2010

[3] P. Zwamborn and P. M. van den Berg, "The three dimensional weak form of the conjugate gradient FFT method for solving scattering problems," IEEE Transactions on Microwave Theory and Techniques, vol. 40, no. 9, pp. 1757-1766, 1992.

[4] S. M. Rao, D. R. Wilton, and A. W. Glisson, "Electromagnetic scattering by surfaces of arbitrary shape," IEEE Transactions on Antennas and Propagation, vol. 30, no. 3, pp. 409-418, 1982.

[5] D. Z. Ding, R.-S. Chen, Z. H. Fan, and P. L. Rui, "A novel hierarchical two-level spectral preconditioning technique for electromagnetic wave scattering," IEEE Transactions on Antennas and Propagation, vol. 56, no. 4, pp. 1122-1132, 2008.

[6] D. Z. Ding, R. S. Chen, and Z. H. Fan, "An efficient SAI preconditioning technique for higher order hierarchical MLFMM implementation," Progress in Electromagnetics Research, vol. 88, pp. 255-273, 2008.

[7] W. C. Chew, J. M. Jin, E. Midielssen, and J. M. Song, Fast and Efficient Algorithms in Computational Electromagnetics, Artech House, Boston, Mass, USA, 2001.

[8] J.Song, "Multilevel fast multipole algorithm for electromagnetic scattering by large complex objects," IEEE Transactions on Antennas and Propagation, vol. 45, no. 10, pp. 1488-1493, 1997.

[9] M. Burton and S. Kashyap, "A study of a recent, momentmethod algorithm that is accurate to very low frequencies," Applied Computational Electromagnetics Society Journal, vol. 10, no. 3, pp. 58-68, 1995.

[10] D. R. Wilton and A. W. Glisson, "On improving the electric field integral equation at low frequencies," in Proceedings of the URSI 
Radio Science Meeting Digest, Los Angeles, Calif, USA, June 1981.

[11] J. R. Mautz and R. F. Harrington, "An e-field solution for a conducting surface small or comparable to the wavelength," IEEE Transactions on Antennas and Propagation, vol. 32, no. 4, pp. 330-339, 1984.

[12] J. S. Lim, S. M. Rao, and D. R. Wilton, "A novel technique to calculate the electromagnetic scattering by surfaces of arbitrary shape," in Proceedings of the URSI Radio Science Meeting Digest, Ann Arbor, Mich, USA, June 1993.

[13] W. Wu, A. W. Glisson, and D. Kajfez, "A comparison of two lowfrequency formulations for the electric field integral equation," in Proceedings of the 10th Annual Review of Progress in Applied Computational Electromagnetics, vol. 2, pp. 484-491, Monterey, Calif, USA.

[14] J.-S. Zhao and W. C. Chew, "Integral equation solution of Maxwell's equations from zero frequency to microwave frequencies," IEEE Transactions on Antennas and Propagation, vol. 48, no. 10, pp. 1635-1645, 2000.

[15] R. J. Adams, "Physical and analytical properties of a stabilized electric field integral equation," IEEE Transactions on Antennas and Propagation, vol. 52, no. 2, pp. 362-372, 2004.

[16] S. Yan, J.-M. Jin, and Z. Nie, "Analysis of electrically large problems using the augmented EFIE with a caldern preconditioner," IEEE Transactions on Antennas and Propagation, vol. 59, no. 6, pp. 2303-2314, 2011.

[17] R. J. Adams and N. J. Champagne II, "A numerical implementation of a modified form of the electric field integral equation," IEEE Transactions on Antennas and Propagation, vol. 52, no. 9, pp. 2262-2266, 2004.

[18] S. H. Christiansen and J.-C. Nédélec, "A preconditioner for the electric field integral equation based on Calderon formulas," SIAM Journal on Numerical Analysis, vol. 40, no. 3, pp. 11001135, 2002.

[19] X.-Q. Sheng and C.-Q. Deng, "A simple and efficient implementation of the well-conditioned electric-field integral equation," IEEE Transactions on Antennas and Propagation, vol. 57, no. 2, pp. 582-586, 2009.

[20] M. B. Stephanson and J.-F. Lee, "Preconditioned electric field integral equation using Calderon identities and dual loop/star basis functions," IEEE Transactions on Antennas and Propagation, vol. 57, no. 4, pp. 1274-1279, 2009.

[21] F. P. Andriulli, K. Cools, H. Bağci et al., "A multiplicative Calderon preconditioner for the electric field integral equation," IEEE Transactions on Antennas and Propagation, vol. 56, no. 8, pp. 2398-2412, 2008.

[22] S. H. Christiansen and J.-C. Nédélec, "A preconditioner for the electric field integral equation based on Calderon formulas," SIAM Journal on Numerical Analysis, vol. 40, no. 3, pp. 11001135, 2002.

[23] H. Chen, J. Zhu, R. S. Chen, and Z. H. Fan, "Fast directional multilevel algorithm combined with Calderon multiplicative preconditioner for stable electromagnetic scattering analysis," Microwave and Optical Technology Letters, vol. 52, no. 9, pp. 1963-1969, 2010.

[24] S. Yan, J.-M. Jin, and Z. Nie, "A comparative study of Calderón preconditioners for PMCHWT equations," IEEE Transactions on Antennas and Propagation, vol. 58, no. 7, pp. 2375-2383, 2010.

[25] L. Greengard, J. Huang, V. Rokhlin, and S. Wandzura, "Accelerating fast multipole methods for the Helmholtz equation at low frequencies," IEEE Computational Science \& Engineering, vol. 5, no. 3, pp. 32-38, 1998.
[26] L. J. Jiang and W. C. Chew, "Low-frequency fast inhomogeneous plane-wave algorithm (LF-FIPWA)," Microwave and Optical Technology Letters, vol. 40, no. 2, pp. 117-122, 2004.

[27] L. J. Jiang and W. C. Chew, "A mixed-form fast multipole algorithm," IEEE Transactions on Antennas and Propagation, vol. 53, no. 12, pp. 4145-4156, 2005.

[28] H. Chen, Z. H. Fan, R. S. Chen, Z. N. Jiang, and M. M. Li, "Adaptive mixed-form fast multipole method for the analysis of electromagnetic scattering," Applied Computational Electromagnetics Society Journal, vol. 25, no. 11, pp. 962-974, 2010.

[29] Z. G. Qian and W. C. Chew, "An augmented electric field integral equation for high-speed interconnect analysis," Microwave and Optical Technology Letters, vol. 50, no. 10, pp. 2658-2662, 2008.

[30] Z.-G. Qian and W. C. Chew, "Fast full-wave surface integral equation solver for multiscale structure modeling," IEEE Transactions on Antennas and Propagation, vol. 57, no. 11, pp. 35943601, 2009.

[31] Z.-G. Qian and W. C. Chew, "Enhanced A-EFIE with perturbation method," IEEE Transactions on Antennas and Propagation, vol. 58, no. 10, pp. 3256-3264, 2010.

[32] Z. Ma, L. Jiang, W. Chew, M. Li, and Z. Qian, "Augmented EPA with augmented EFIE method for packaging analysis," in Proceedings of the IEEE Electrical Design of Advanced Packaging and Systems Symposium (EDAPS '10), December 2010.

[33] Y. P. Chen, L. Jiang, Z.-G. Qian, and W. C. Chew, "An augmented electric field integral equation for layered medium Green's function," IEEE Transactions on Antennas and Propagation, vol. 59, no. 3, pp. 960-968, 2011.

[34] J. Shaeffer, "Direct solve of electrically large integral equations for problem sizes to $1 \mathrm{M}$ unknowns," IEEE Transactions on Antennas and Propagation, vol. 56, no. 8, pp. 2306-2313, 2008.

[35] K. Zhao, M. Vouvakis, and J.-F. Lee, "The adaptive cross approximation algorithm for accelerated method of moments computations of EMC problems," IEEE Transactions on Electromagnetic Compatibility, vol. 47, no. 4, pp. 763-773, 2005.

[36] Z. N. Jiang, R. S. Chen, Z. H. Fan, Y. Y. An, M. M. Zhu, and K. W. Leung, "Modified adaptive cross approximation algorithm for analysis of electromagnetic problems," Applied Computational Electromagnetics Society Journal, vol. 26, no. 2, pp. 160-169, 2011.

[37] D. Gope and V. Jandhyala, "Efficient solution of EFIE via lowrank compression of multilevel predetermined interactions," IEEE Transactions on Antennas and Propagation, vol. 53, no. 10, pp. 3324-3333, 2005.

[38] K. A. Michalski and D. Zheng, "Electromagnetic scattering and radiation by surfaces of arbitrary shape in layered media, II: implementation and results for contiguous half-spaces," IEEE Transactions on Antennas and Propagation, vol. 38, no. 3, pp. 345-352, 1990.

[39] M. I. Aksun, "A robust approach for the derivation of closedform green's functions," IEEE Transactions on Microwave Theory and Techniques, vol. 44, no. 5, pp. 651-658, 1996.

[40] Z. Jiang, R.-S. Chen, Z. Fan, and Y. An, "Modified compressed block decomposition preconditioner for electromagnetic problems," Microwave and Optical Technology Letters, vol. 53, no. 8, pp. 1915-1919, 2011.

[41] Z. N. Jiang, Z. H. Fan, D. Z. Ding, R. S. Chen, and K. W. Leung, "Preconditioned MDA-SVD-MLFMA for analysis of multi-scale problems," Applied Computational Electromagnetics Society Journal, vol. 25, no. 11, pp. 914-925, 2010.

[42] C. Alex Woo, T. G. Helen Wang, and J. Michael Schuh, "Benchmark radar targets for the validation of computational 
electromagnetics programs," IEEE Transactions on Antennas and Propagation, vol. 35, no. 1, pp. 84-89, 1993.

[43] J. Wu, S. N. Qiu, C. X. Qiu, and I. Shih, "Wideband microstrip bandpass filter based on intercoupled split-ring resonator," Microwave and Optical Technology Letters, vol. 49, no. 8, pp. 1809-1813, 2007.

[44] J.-X. Chen, T. Y. Yum, J.-L. Li, and Q. Xue, "Dual-mode dual-band bandpass filter using stacked-loop structure," IEEE Microwave and Wireless Components Letters, vol. 16, no. 9, pp. 502-504, 2006. 

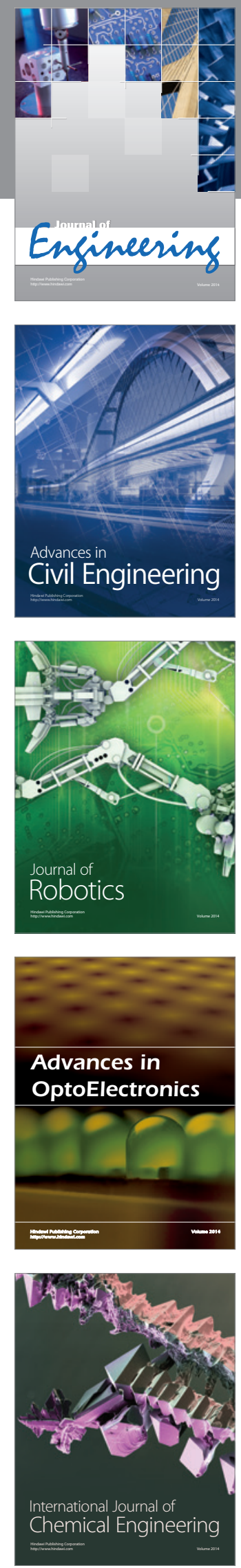

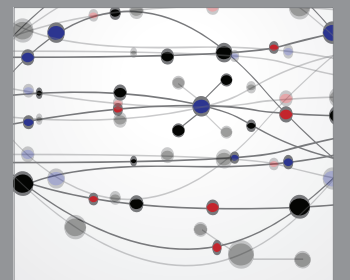

The Scientific World Journal
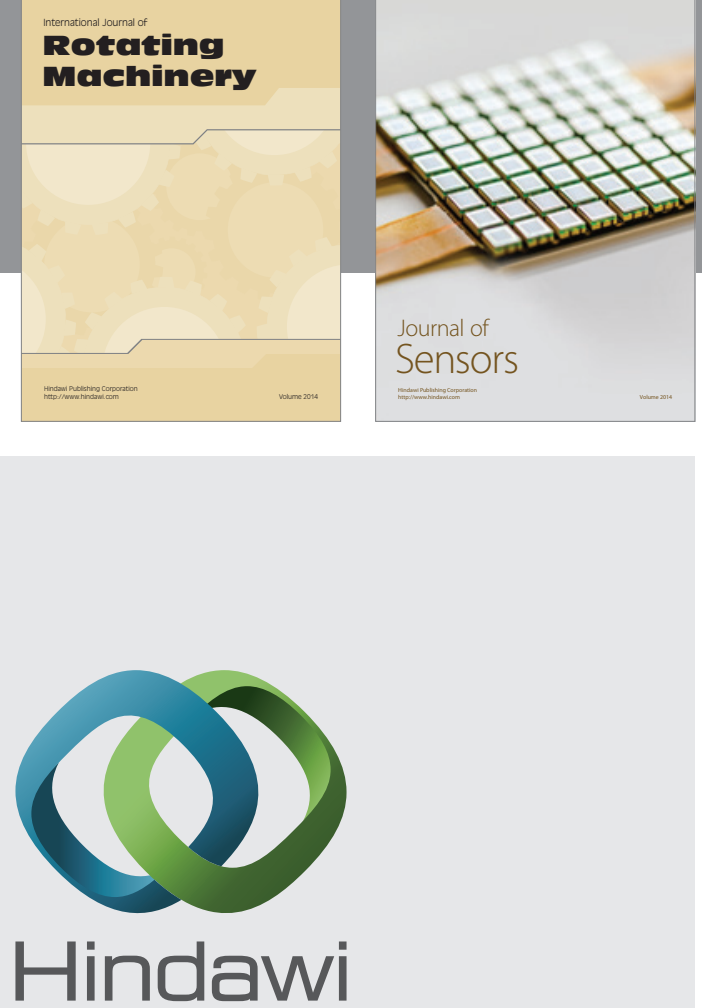

Submit your manuscripts at http://www.hindawi.com
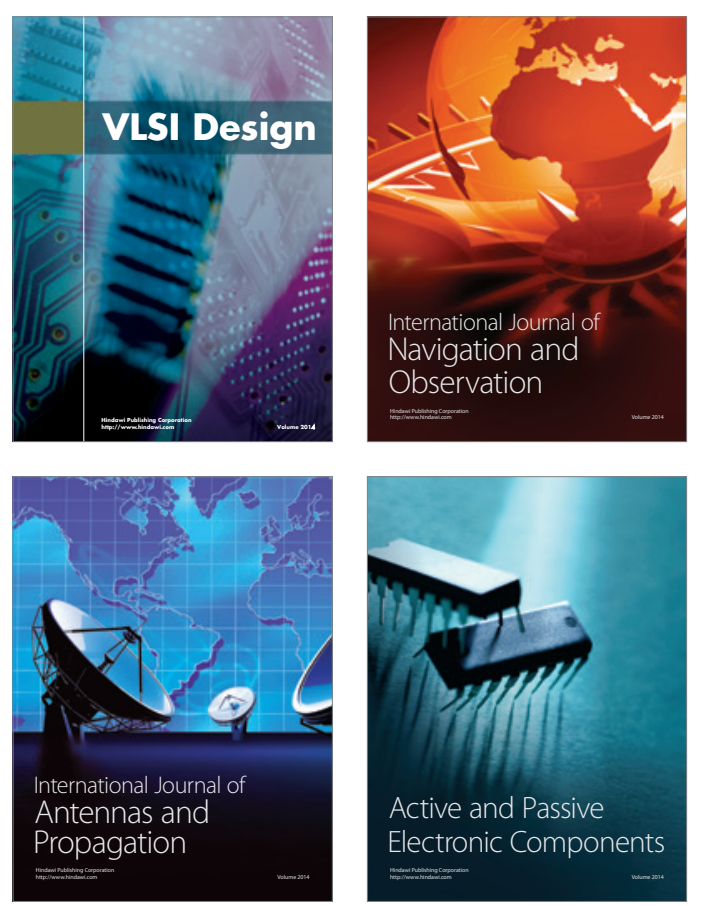
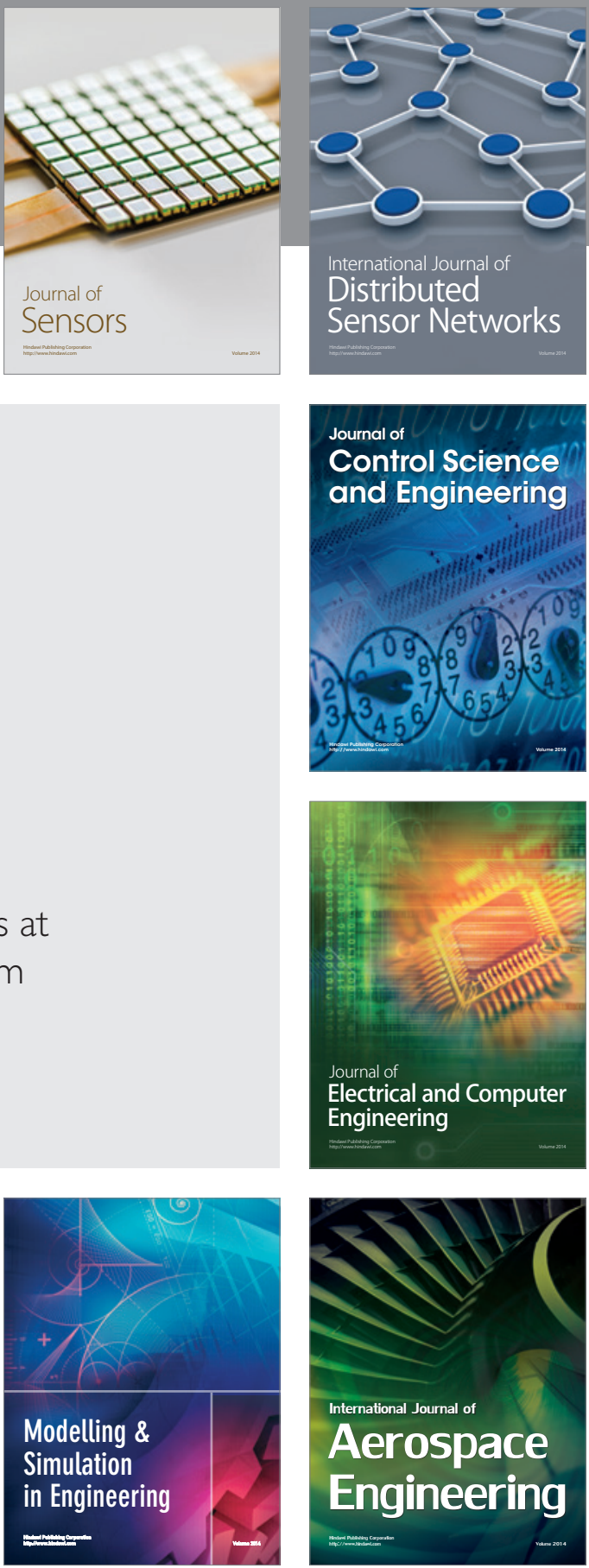

Journal of

Control Science

and Engineering
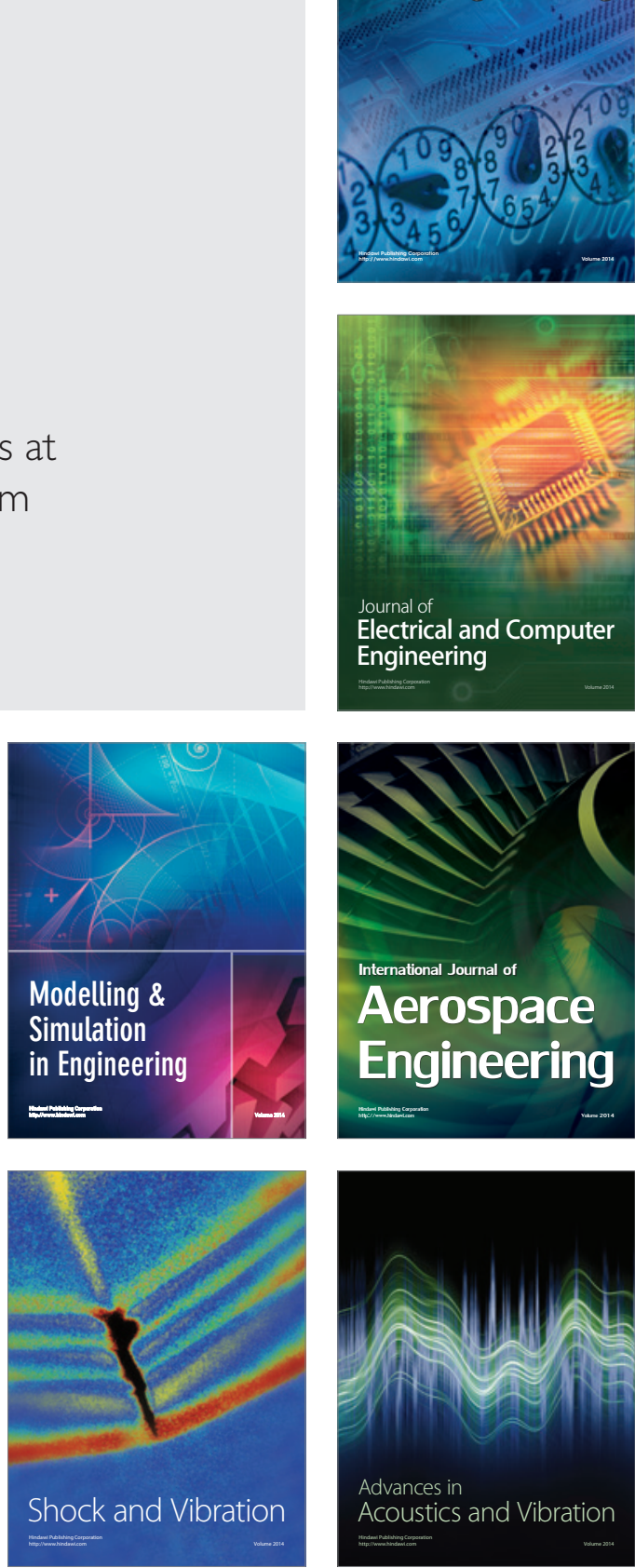